\title{
Flow Cytometric Method for Estimation of 5-bromo-2'-deoxyuridine Content in Rat Serum
}

\author{
J. MIKEŠ ${ }^{1}$, J. ŠEVC ${ }^{1}$, J. KOŠUTH ${ }^{1}$, A. MATIAŠOVÁ ${ }^{1}$, Z. DAXNEROVÁ ${ }^{1}$, \\ P. FEDOROČKO ${ }^{1}$ \\ ${ }^{1}$ Institute of Biology and Ecology, Faculty of Sciences, P. J. Šafárik University in Košice, Košice, \\ Slovakia
}

Received February 14, 2014

Accepted May 6, 2014

On-line August 26, 2014

\begin{abstract}
Summary
Labelling of DNA in replicating cells using 5-bromo-2'deoxyuridine (BrdU) is widely used, however the rapid clearance and metabolisation of BrdU in the living organism is a critical issue. Although the pharmacokinetic of BrdU in experimental animals is empirically approximated, the exact time-curve remains unknown. Here we present novel method for estimation of the BrdU content in the blood serum. The application is based on the in vitro cocultivation of tumour cells with the examined serum and the subsequent quantification of the incorporated BrdU in the DNA using flow cytometry analysis. Our results demonstrate that this approach can quantify the BrdU concentration in serum at $1 \mu \mathrm{mol} \cdot \mathrm{dm}^{-3}$ and might represent an attractive alternative to conventional chromatographic analysis. The employment of tumour cells as "detectors" of the BrdU content in serum provides an advantage over high pressure liquid chromatography (HPLC), as this approach allows us to approximate not only the concentration of $\mathrm{BrdU}$, but also to determine, whether BrdU is present in the blood serum in effective concentration to reliable label all cells undergoing the S-phase of the cell cycle. The presented application might be a helpful tool for studies on pharmacokinetics of BrdU or other thymidine analogues when testing various administration routes or protocols.
\end{abstract}

\section{Key words}

BrdU pharmacokinetic • BrdU incorporation - Cell proliferation • Flow cytometry $\bullet$ HCT-116

\section{Corresponding author}

J. Ševc, Institute of Biology and Ecology, Faculty of Science, P. J. Šafárik University in Košice, Moyzesova 11, 04154 Košice, Slovakia. E-mail: juraj.sevc@upjs.sk

\section{Introduction}

The identification of DNA-synthesising cells using halogenated analogues of thymidine, such as 5-bromo-2'-deoxyuridine (bromodeoxyuridine, BrdU), is a well-established procedure. In addition to highly specific applications, such as the evaluation of the S-phase transition or S-phase arrest (Dolbeare et al. 1983), DNA repair (Selden et al. 1994), abortive cell cycle re-entry (Bauer and Patterson 2005), or gene duplication, BrdU is the most widely used technique to label proliferating cells or to track their progeny (for a review see Taupin 2007). The incorporation of BrdU can be visualized using light and fluorescent microscopy, or it can be measured by fluorescence detectors and flow cytometers. Because BrdU crosses the blood-brain barrier, it can be delivered, even in adult neurogenesis studies, by intracerebroventricular (Zhao and Janson Lang 2009), intraperitoneal (Ševc et al. 2011, Kútna et al. 2014), and intravenous injection (Phuphanich and Levin 1985), or by oral administration (Spassky et al. 2005). However, the exact pharmacokinetic properties of BrdU administered by different routes are unknown (Taupin 2007). It is estimated that BrdU is available for labelling for approximately $2 \mathrm{~h}$ after a systemic injection, and then its levels drop rapidly (Packard et al. 1973, Hayes and 
Nowakowski 2000). To ensure both, the reliable labelling of proliferating cells and proper interpretation of obtained results, it is important to know the pharmacokinetic properties of BrdU. Chromatographic methods, such as high pressure liquid chromatography (HPLC), offer a highly sensitive and quantitative approach, but they do not take into consideration the attributes of biological systems, such as "permeability" of cellular membranes for BrdU, or the molar ratio of BrdU and thymidine. With respect to disadvantages of chromatographic methods, Barker et al. (2013) presented simply and inexpensive method for detection of BrdU in blood serum. Their method is based on in vitro cocultivation of eukaryotic cells adhered on glass coverslips with sera containing BrdU to allow the cells to incorporate the BrdU from the serum. Subsequently, incorporated BrdU is visualized with standard immunocytochemical staining techniques and semiquantitatively evaluated under light microscope. On the one hand, this method enables to detect the BrdU in concentrations suitable for labelling of S-phase cells in blood serum, but on the other hand, employment of light microscopic analyses might be resulted in somewhat truncated results.

For these reasons, here we report flow cytometric method for estimation of the BrdU content in rat serum using a simple approach based on the cultivation of rapidly proliferating tumour cells with serum isolated from the blood of experimental animals. We demonstrated that i) the intensity of staining evaluated as the fluorescence median corresponds to the concentration of $\mathrm{BrdU}$ in the serum, and more importantly ii) the percentage of BrdU-labeled S-phase cells determines, whether BrdU is present in the blood serum in effective concentration for reliable labelling all cells undergoing the S-phase of the cell cycle. Therefore this method might be used in BrdU pharmacokinetic studies. Data demonstrating the applicability of the method under in vivo conditions, as well as comparison of our method with conventional HPLC method, are presented too.

\section{Methods}

\section{Collection of blood sera and preparation of the BrdU molar concentration scale}

The experimental procedures were performed with approval from the National Veterinary and Food Administration of the Slovak Republic and the Animal Care Committee of Pavol Jozef Šafárik University in
Košice, Slovak Republic, in accordance with the European Communities Council Directive 86/609/EEC and in compliance with current national legislation. Intact adult Wistar albino rats of both sexes were used in this study. Each animal had ad libitum access to food and water and was fed on a complete and balanced standard laboratory diet (Peter Misko, Snina, Slovak Republic). The animals were sacrificed by cervical decapitation under terminal anesthesia; the trunk blood was collected, allowed to clot, and centrifuged to obtain clear serum. The serum from several animals $(n=14)$ was pooled, filtered, and spiked with BrdU (Sigma-Aldrich, Steinheim, Germany) at the following molar concentrations: $20,15,10,5,1,0.5$, and $0.1 \mu \mathrm{mol} . \mathrm{dm}^{-3}$ and deeply frozen $\left(-80^{\circ} \mathrm{C}\right)$ for at least one week to mimic the standard procedure required for sample processing.

Confirmation of the presented method in vivo was executed as follows. A single dose of BrdU (i.p., $100 \mathrm{mg} / \mathrm{kg}$ ) was administered to adult rats weighing between 290 and $310 \mathrm{~g}$. The animals were sacrificed by cervical decapitation $45(n=3)$ and $240 \min (n=3)$ after injection. The trunk blood was collected and processed to obtain clear sera. The serum samples were deeply frozen until the beginning of the experiment.

\section{Adhesion cell culture}

Colon adenocarcinoma HCT-116 cells (ATCC\# CCL-247, ATCC, Rockville, MD, USA) were cultured in McCoy's 5A medium (Sigma-Aldrich, St. Louis, MO, USA) supplemented with $10 \%$ fetal calf serum (Gibco Invitrogen Corp., Carlsbad, CA, USA), antibiotics (penicillin $100 \mathrm{Uml}^{-1}$, streptomycin $100 \mathrm{mg} \cdot \mathrm{ml}^{-1}$, and

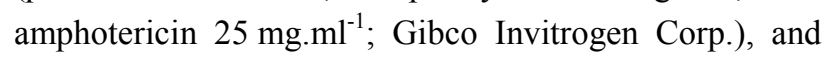
gentamycin $\left(50 \mathrm{mg} \mathrm{ml}^{-1}\right.$; Sigma-Aldrich). The cultures were maintained at $37{ }^{\circ} \mathrm{C}$ in $5 \% \mathrm{CO}_{2}$ and $95 \%$ humidity. For the experiments, the cells were seeded in 6-well $\left(6 \times 10^{5}\right.$ cells per well) tissue culture plates (TPP, Trasadingen, Switzerland) at an approx. $50 \%$ density to eliminate proximity inhibition that is typical of overgrown cell cultures and thus ensure their rapid proliferation in the log-phase. The cells were allowed to settle for $24 \mathrm{~h}$, and then the medium was replaced with $1.5 \mathrm{ml}$ of rat serum containing $\mathrm{BrdU}(20,10,5,1,0.5$, $0.1 \mu \mathrm{mol} . \mathrm{dm}^{-3}$ ) or rat serum from the experimental animals, and the cells were cultivated for 1 or $4 \mathrm{~h}$. Afterwards, the serum was discarded; the cells were washed with phosphate buffered saline (PBS), harvested by trypsinisation, washed once in PBS, and further 
processed according to the BrdU/PI staining protocol.

\section{BrdU/PI staining protocol}

After washing the cells in PBS, the supernatant was discarded; the cells were fixed in ice-cold $70 \%$ ethanol and stored at $-20{ }^{\circ} \mathrm{C}$ until further processing. The collected samples were processed as follows: the cells were centrifuged, and the ethanol was discarded; then, the cells were washed in PBS; the DNA was denaturated in $2 \mathrm{M} \mathrm{HCl}$ for $1 \mathrm{~h}$ at $37^{\circ} \mathrm{C}$; the cells were centrifuged and resuspended in $0.1 \mathrm{M}$ sodium borate $(\mathrm{pH}=8.5)$ for $10 \mathrm{~min}$ at room temperature (RT); then, the cells were centrifuged and washed in PBS; permeabilized with $0.1 \%$ Tween- $20+1 \%$ BSA in PBS for 5 min at RT; washed two times with $1 \%$ BSA in PBS; split into halves; incubated with the anti-BrdU antibody or an isotype control (BD Pharmingen, Franklin Lanes, NJ, USA; cat\# 556028) in $1 \%$ BSA in PBS for $30 \mathrm{~min}$ at RT, and washed two times in $1 \%$ bovine serum albumin (BSA) in PBS. The RNA was degraded with ribonuclease A (Sigma-Aldrich), and the nuclear DNA was stained with propidium iodide (PI, $20 \mu \mathrm{g} . \mathrm{ml}^{-1}$; Sigma-Aldrich) in Vindelov's solution at RT for $30 \mathrm{~min}$. Finally, the cells were analyzed using a BD FACSCalibur flow cytometer (Becton Dickinson, San Jose, CA, USA) in standard setup with one excitation $488 \mathrm{~nm} 15 \mathrm{~mW}$ argon laser and three fluorescent channels (FL1 - 530/30; FL2 - 582/42; FL3 - >670).

\section{Data analysis details}

An acquisition gate was established based on the FSC x SSC, propidium iodide fluorescence (FL2-channel) width versus area (FL2-W x FL2-A) and FL2-A x FSC to exclude debris and doublets. All flow cytometry measurements were performed on 10,000 cells per sample. Using flow cytometry analyses, two BrdU pharmacokinetics parameters were evaluated, the staining index (SI) and the percentage of BrdU-positive S-phase cells. The SI represents the amount of BrdU incorporated in the newly synthetized DNA of S-phase cells and reflects the relative concentration of BrdU in the blood serum at each time-point. The SI was calculated as the ratio of the median fluorescence intensity (MFI) of S-phase cells that were positively stained for BrdU and the MFI of G2/M cells from the iso-type control. The percentage of BrdU-positive S-phase cells reflects whether the concentration of $\mathrm{BrdU}$ at a certain time-point is sufficient for reliable labelling of all cells undergoing the S-phase of the cell cycle. The percentage of BrdU- positive S-phase cells was calculated as the ratio of BrdU-positive S-phase cells to all S-phase cells. In both analyses, each time-point was represented as the mean \pm SEM calculated from three samples. BD CellQuest Pro v3.3 (Becton Dickinson) software was used to analyse the data; FlowJo software, ver. 7.6.5 (Tree Star, Inc., Ashland, OR, USA) was used to generate the graphical output. The images were assembled into figures using Adobe Illustrator (Adobe Systems, Inc., San Jose, USA).

\section{HPLC analysis}

BrdU was dissolved in redistilled water or in intact rat blood serum to obtain internal standards $\left(20 \mu \mathrm{mol} \cdot \mathrm{dm}^{-3}\right)$. Prior to HPLC analyses, the samples containing rat serum (internal standard or serum from experimental animals) were filtered through a $0.2-\mu \mathrm{m}$ filter, thoroughly mixed with an equal amount of chloroform for $1 \mathrm{~min}$, and centrifuged at $18000 \mathrm{G}$ for $5 \mathrm{~min}$. The top layer (deproteinised serum) was collected and subjected to HPLC analysis. The separation was performed with a Nucleodur PolarTec $125 \times 4.0 \mathrm{~mm}$, $5 \mu \mathrm{m}$ column (Macherey-Nagel, Dueren, Germany) maintained at $30{ }^{\circ} \mathrm{C}$ under isocratic flow conditions. The injection volume was $25 \mu$ l. The mobile phase was $2 \%$ methanol in $0.01 \mathrm{M}$ potassium phosphate $(\mathrm{pH} \mathrm{3.5)}$ at a flow rate of $0.65 \mathrm{ml} \cdot \mathrm{min}^{-1}$. The BrdU was identified and quantified by an Agilent Infinity 1260 HPLC system (Agilent Technologies, Palo Alto, USA) equipped with a diode array detector according to the retention time and the absorption spectra of the standard. The BrdU was monitored at $280 \mathrm{~nm}$ wavelength at $17.5 \mathrm{~min}$ retention time.

\section{Statistical analysis}

The results were tested using one-way ANOVA with the Student-Newman-Keuls post-hoc method. Differences between individual time-points or groups were considered statistically significant if $* \mathrm{P}<0.05$, $* * \mathrm{P}<0.01$ and $* * * \mathrm{P}<0.001$.

\section{Results}

Adherent colon adenocarcinoma HCT-116 cells were seeded and treated according to the protocol described above under optimal conditions to ensure their rapid proliferation in the log-phase. First, one hour incubation of HCT-116 cells with sera containing BrdU was tested. The gating strategy and an example of the 
analysis are presented (Fig. 1). Each sample was divided into halves and stained separately with an anti-BrdU antibody (Fig. 1a) or with an iso-type control (Fig. 1b). Only the cells that were sequentially gated as stated above (Gates 1-3, Fig. 1) were subjected to further analyses. The staining index (SI) was calculated as a ratio of the median fluorescence intensity (MFI) of S-phase cells positively stained for BrdU (S BrdU+ gate, Fig. 1a) and MFI of $\mathrm{G}_{2} / \mathrm{M}$ cells of the iso-type control (G2/M gate, Fig. 1b). Therefore the concentrations with SI equal to 1 (or lower) are below the detection limit of our method. To determine, whether the presented approach is sensitive to various concentrations of $\mathrm{BrdU}$ in the rat serum, a series of analyses using sera spiked with known BrdU concentrations $(0.1,0.5,1,5,10,15$, and $20 \mu$ mol. $\mathrm{dm}^{-3}$ ) were performed. The means \pm SEM of three samples are presented. As seen, the intensity of staining is clearly BrdU-concentration dependent (Fig. 2a). Statistical analysis confirmed the significance $(\mathrm{p}=0.002)$ of the concentration-dependent BrdU incorporation. According to obtained results, our approach can quantify the BrdU concentration in serum at $1 \mu$ mol.dm ${ }^{-3}$. Employment of flow cytometry analysis of BrdU-labelled cells enables to focus on the S-phase cells in order to determine the effective concentration of BrdU in the blood serum. In the next step, the percentage of
BrdU-labeled S-phase cells was tested across the concentration curve. The percentage of BrdU labelled cells was calculated as the ratio of BrdU-positive S-phase cells (S BrdU+ gate) to all S-phase cells (sum of S BrdU+ and S BrdU- gates, Fig. 1a). Similarly as with SI index, our method can record the presence of BrdU in the blood serum in concentration $\geq 1 \mu$ mol.dm ${ }^{-3} \quad(p=0.0266$ in comparison with negative control, Fig. 2b). Moreover, tumour cells responds to increasing concentration of BrdU also with increasing percentage of BrdU-labelled S-phase cells. According to our findings, BrdU labels reliably all S-phase cells in concentrations $\geq 20 \mu \mathrm{mol} . \mathrm{dm}^{-3}$.

To compare our BrdU detection method with conventional HPLC analysis, we tested the detection limit of HPLC for BrdU dissolved in redistilled water and in intact rat serum according to previously described protocols (Russo et al. 1984, DeFazio et al. 1987). According to our results, HPLC method was able to detect and quantify as much as $19 \mathrm{ng}$ of BrdU dissolved in water, i.e. $2.5 \mu \mathrm{mol} . \mathrm{dm}^{-3}$ solution at a $25 \mu$ injection volume. However, the detection limit of BrdU in the serum was lower $\left(6.4 \mu \mathrm{mol} . \mathrm{dm}^{-3}\right)$. The presence of an unidentified compound with a similar retention time and spectral characteristics as BrdU hindered the higher resolution of our detection.
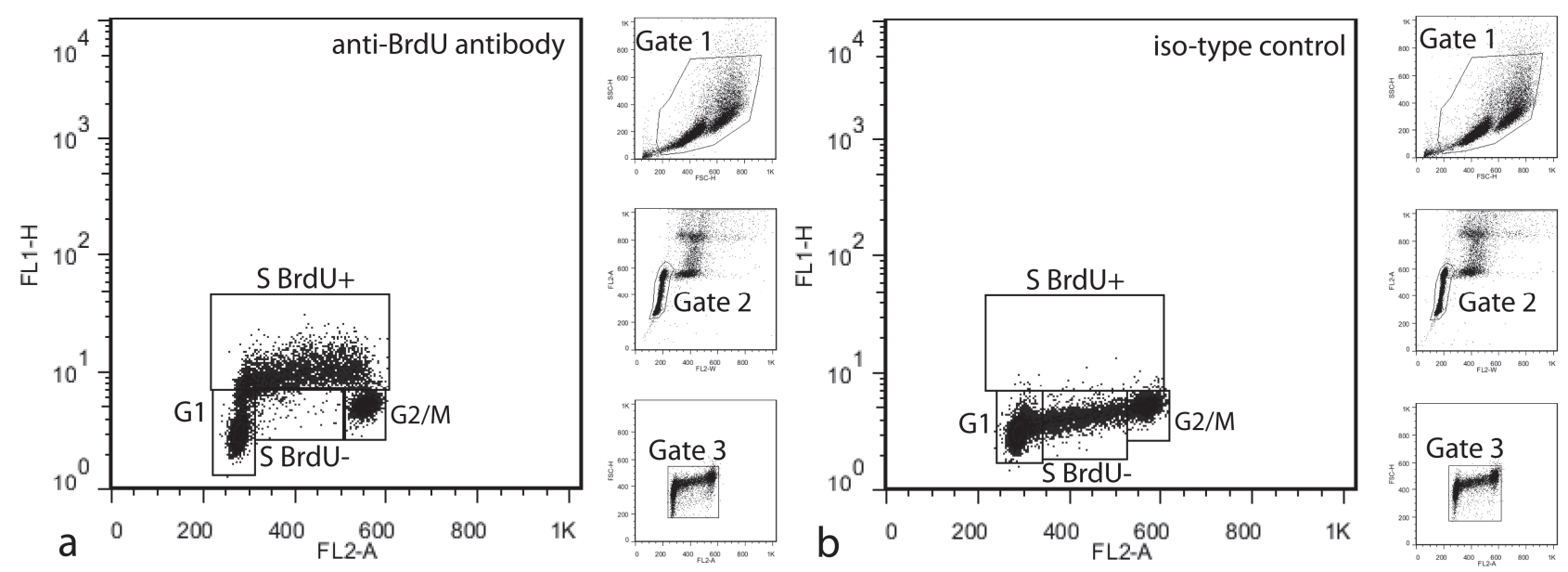

Fig. 1. Schematic representation of the data analysis strategy. Each sample was halved and stained with an anti-BrdU antibody (a) or with an iso-type control antibody (b). The cells were gated to eliminate the debris and cell doublets. The debris was eliminated based on the physical attributes of the measured events by "Gate 1" on the FSC $x$ SSC plot (event size $x$ internal integrity/granularity). Cell doublets, characterized by wider fluorescence of the peak staining for DNA content and a higher size, were eliminated based on the DNA content (FL2-A channel) and the width of the fluorescent peak (FL2-W channel) or the size of the event (FSC) by "Gate 2" on the FL2-W x FL2-A plot and by "Gate 3" on the FSC x FL2-A plot, respectively. Events that passed through a positive combination of gates 1-3 were further analyzed in the FL2-A x FL1-H (BrdU staining) plot. The staining index was calculated as the proportion of the medians of BrdU-positive cells stained with the anti-BrdU antibody ( $\mathrm{a}, \mathrm{S}$ BrdU+ gate) to the $\mathrm{G}_{2} / \mathrm{M}$-phase cells stained with an iso-type control antibody ( $b, G 2 / M$ gate). The percentage of BrdU labelled cells was calculated as the ratio of BrdU-positive S-phase cells (a, S BrdU+ gate) to all S-phase cells (a, sum of S BrdU+ and S BrdU- gates). 

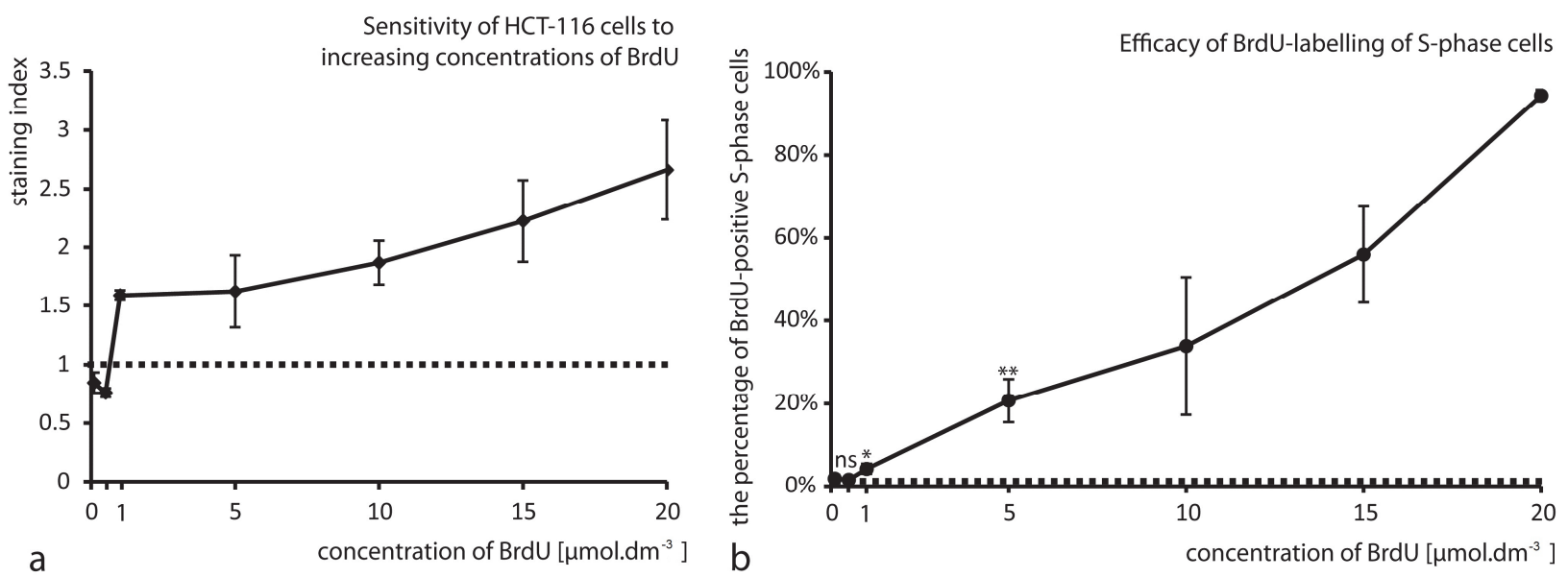

Fig. 2. Quantitative analysis of BrdU incorporation in relation to its serum concentration. HCT-116 cells were cultivated for $1 \mathrm{~h}$ with serum containing $\operatorname{BrdU}\left(0.1,0.5,1,5,10,15\right.$ and $20 \mu \mathrm{mol}_{\mathrm{dm}} \mathrm{dm}^{-3}$ and then processed and analyzed according to the standard protocol. The results are presented as the mean \pm SEM of three samples. In the experiment, the dependency of staining index (a) and percentage of S-phase BrdU-labelled cells (b) on the increasing concentration of BrdU was investigated. Dotted line - detection limit of presented method was $\leq 1$ (a) or equal or lower than the negative control (b); ns - non significant, $*-p<0.05, * *-p<0.01$ in comparison with negative control.

As a verification of the in vivo applicability of our results, the content of $\mathrm{BrdU}$ in rat serum after i.p. administration of a single dose of BrdU $(100 \mathrm{mg} / \mathrm{kg}$ of body weight) at two different time-points was analyzed. According to empirical experience and the results of HPLC analyses, it is estimated that BrdU persists in the blood serum after i.p. injection for approximately $2 \mathrm{~h}$ (Packard et al. 1973, Phuphanich and Levin 1985, Taupin 2007). Therefore, the concentration of BrdU in rat serum should be high enough to be detectable $45 \mathrm{~min}$ after i.p. injection. According to our results, analysis of SI revealed that BrdU should be in slightly higher concentration than $20 \mu \mathrm{mol} . \mathrm{dm}^{-3}$ (Fig. 3). HPLC quantification revealed that the concentration of $\mathrm{BrdU}$ in rat serum 45 min after administration was $24.33 \pm 0.78$ $\mu$ mol. $\mathrm{dm}^{-3}$. More importantly, analysis of the percentage of BrdU-labeled S-phase cells revealed that BrdU is in effective concentration for reliable labelling of S-phase cells $(94.4 \pm 2.1 \%)$ in the rat serum 45 min after i.p. injection. On the other hand, the concentration of BrdU in the serum at the 240 min time-point was below the detection limit of both our flow cytometric method and HPLC.

Finally, a prolonged 4-h incubation was tested (data not shown), but this did not increase the sensitivity of the presented method. Nevertheless, the higher percentage of positively stained cells demonstrated that labelling an entire asynchronous cell population requires a longer exposure to BrdU.

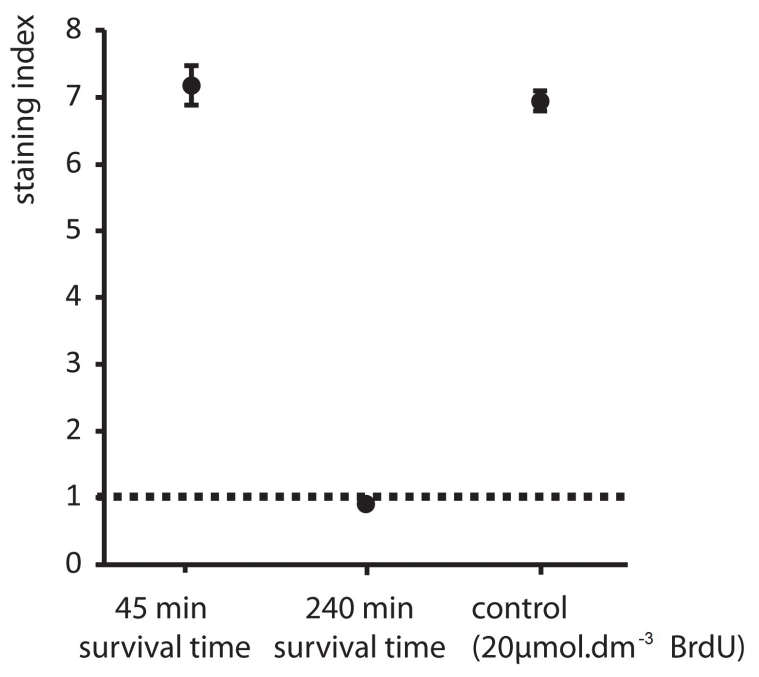

Fig. 3. The presence of BrdU in rat serum after i.p. injection. The experimental animals were i.p. injected with $\mathrm{BrdU}(100 \mathrm{mg} / \mathrm{kg}$ dose) and sacrificed 45 or 240 min afterwards. HCT-116 cells were cultivated for $1 \mathrm{~h}$ with sera isolated from experimental animals and then processed and analyzed according to the standard protocol. The results are presented as the mean \pm SEM of three samples. As the positive control, blood serum spiked with $\mathrm{BrdU}$ at $20 \mu \mathrm{mol} . \mathrm{dm}^{-3}$ concentration was used. Dotted line detection limit of flow cytometric method.

\section{Discussion}

According to our experimental design, BrdU was used to label proliferating tumour cells. Rather than determining the proliferation rate of the cells, we sought to detect the level of $\mathrm{BrdU}$ in the serum that was accessible by the cells. Similar method 
invented by Barker et al. (2013) employed classic immunohistochemical detection of BrdU coupled with light microscopy and semiquantitative analysis. In our experiment, flow cytometry was used, because in contrast to microscopic or other analytical methods, it enables fast and reliable multiparametric analyses (Hoshino et al. 1985) on a cell-to-cell basis. Moreover, focusing on the S-phase cell fraction during the analyses allows to calculate the percentage of BrdU-positive S-phase cells and to determine the effective (target) concentration for reliable labelling of all S-phase cells. From a practical point of view, it seems that this parameter may be even more important than simple determination of BrdU concentration in the blood serum. This could be true in unnecessarily high doses of BrdU (e.g. more than $20 \mu \mathrm{mol} . \mathrm{dm}^{-3}$ ) with respect to cytostatic and cytotoxic side-effects of BrdU (Kuan et al. 1996, Kuhn and Cooper-Kuhn 2007).

Although our results prove that the intensity of staining with an anti-BrdU antibody correspond to the serum level, it was concluded that concentrations equal to or less than $1 \mu \mathrm{mol} . \mathrm{dm}^{-3}$ are not sufficient for contrastive staining of proliferating cells. Moreover, during the experiment we noticed that the percentage of positively stained cells is even more dependent on BrdU concentration than the staining index (e.g. standard errors at 10 and $15 \mu \mathrm{mol} . \mathrm{dm}^{-3}$ BrdU concentrations in Figure 2b). These findings raise an important issue. If the labelling of proliferating cells with BrdU is dependent on the BrdU concentration, then the identification of proliferating cells could be highly variable, especially when the concentration of BrdU in the serum drops below the optimal range. Therefore, we presume that a deeper knowledge of pharmacokinetic properties of BrdU in vivo is needed. Our results also indicate that even if the BrdU concentration in the serum could be theoretically detectable by even more sensitive analytic methods, it does not necessarily correspond to detectable staining of S-phase cells. On the other side, it is important to mention that the purpose of the presented method is to offer an alternative tool for the approximation of BrdU pharmacokinetics in serum, but not to provide an accurate concentration measurement.

\section{Comparison with existing methods}

To this date, most relevant methods for determination of BrdU concentration in blood serum are HPLC (e.g. Kriss et al. 1963, Russo et al. 1984, Phuphanich and Levin 1985, Kuan et al. 1996) and in vitro bioassay reported in Barker et al. (2013). HPLC is purely quantitative analytical method with most reproducible and reliable results. Moreover it is fast, modest and inexpensive method. On the other hand, as mentioned previously, knowledge on the exact concentration of BrdU in blood serum may be insufficient under certain circumstances. In our experiment, we performed HPLC analyses according to well-established protocols (Russo et al. 1984, DeFazio et al. 1987). Surprisingly, in contrast to our flow cytometric method, the detection limit of HPLC was lower and the presence of unidentified metabolite in the blood sera of experimental animals with similar spectral characteristics and retention time even hindered the quantification of BrdU concentration. In the other hand, in vitro bioassay reported in Barker et al. (2013) employs similar approach as our method. Cocultivation of eukaryotic cells adhered on glass coverslips with sera containing BrdU, followed by conventional immunocytochemical detection of BrdU and light microscopic analysis predicts strengths and weaknesses of this approach. First, it is somewhat easier method with rapid results and requires less volume of the blood serum for the in vitro cocultivation with cells. On the contrary, flow cytometric analysis enables multiparametric analysis of thousands of cells with focus on S-phase fraction. Moreover, since BrdU pharmacokinetics and its uptake by cells (both in in vivo and in vitro conditions) seem to be intricate, our presented approach may represent the efficient tool for its solving.

\section{Applicability of the flow-cytometric method}

Regarding the application, the presented approach might help, for example, to evaluate the BrdU pharmacokinetics with various modes of administration (unpublished results) and thus also to test various concentrations with respect to the quest for optimal non/low-toxic doses. It might be also a helpful approach to study the various strategies for the alteration of BrdU pharmacokinetics or to test a potential effect of experimental drugs/their metabolites on BrdU internalisation, as it can drift the estimation of proliferating cells in vivo and lead to erroneous conclusions on its mechanisms. Because the BrdU dosing used in various experiments was only empirically approximated and unnecessarily high doses might be toxic, rationalisation could lead to the suppression of side effects. Although the experiment was conducted with rat serum, it is probable that this method might be applied 
for the analysis of serum originating from other experimental animals or humans.

\section{Conflict of Interest}

There is no conflict of interest.

\section{Acknowledgements}

This work was supported by the Slovak Research and
Development Agency under the contract No. VVCE0001-07, by the Scientific Grant Agency of the Ministry of Education of the Slovak Republic No. VEGA 1/0322/11 and 1/0967/12 and by structural funds of EU under contract No. 007/20092.1/OPVaV. The authors are grateful to Viera Balážová and Eva Pástorová for assistance with technical procedures.

\section{References}

BARKER JM, CHARLIER TD, BALL GF, BALTHAZART J: A new method for in vitro detection of bromodeoxyuridine in serum: A proof of concept in a songbird species, the Canary. PLoS One 8: 1-5, 2013.

DEFAZIO A, LEARY JA, HEDLEY DW, TATTERSALL MHN: Immunohistochemical detection of proliferating cells in vivo. J Histochem Cytochem 35: 571-577, 1987.

DOLBEARE F, GRATZNER H, PALLAVICINI MG, GRAY JW: Flow cytometric measurement of total DNA content and incorporated bromodeoxyuridine. Proc Natl Acad Sci USA 80: 5573-5577, 1983.

HAYES NL, NOWAKOWSKI RS: Exploiting the dynamics of S-phase tracers in developing brain: interkinetic nuclear migration for cells entering versus leaving the S-phase. Dev Neurosci 22: 44-55, 2000.

HOSHINO T, NAGASHIMA T, MUROVIC J, LEVIN EM, LEVIN VA, RUPP SM: Cell kinetic studies of in situ human brain tumors with bromodeoxyuridine. Cytometry 6: 627-632, 1985.

KRISS JP, MARUYAMA Y, TUNG LA, BOND SB, REVESZ L: The fate of 5-bromodeoxyuridine, 5-bromodeoxycytidine, and 5-iododeoxycytidine in man. Cancer Res 23: 260-268, 1963.

KUAN HY, SMITH DE, ENSMINGER WD, KNOL JA, DEREMER SJ, YANG Z, STETSON PL: Regional pharmacokinetics of 5-bromo-2'-deoxyuridine and 5-fluorouracil in dogs: Hepatic arterial versus portal venous infusions. Cancer Res 56: 4724-4727, 1996.

KUHN HG, COOPER-KUHN CM: Bromodeoxyuridine and the detection of neurogenesis. Curr Pharm Biotechnol 8: 127-131, 2007.

KÚTNA V, ŠEVC J, GOMBALOVÁ Z, MATIAŠOVÁ A, DAXNEROVÁ Z: Enigmatic cerebrospinal fluid-contacting neurons arise even after the termination of neurogenesis in the rat spinal cord during embryonic development and retain their immature-like characteristics until adulthood. Acta Histochem 116: 278-285, 2014.

PACKARD DS JR, MENZIES RA, SKALKO RG: Incorportaiton of thymidine and its analogue, bromodeoxyuridine, into embryos and maternal tissues of the mouse. Differentiation 1: 397-404, 1973.

PHUPHANICH S, LEVIN VA: Bioavailability of bromodeoxyuridine in dogs and toxicity in rats. Cancer Res $\mathbf{4 5}$ : 2387-2389, 1985.

RUSSO A, GIANNI L, KINSELLA TJ, KLECKER RW JR, JENKINS J, ROWLAND J, GLATSTEIN E, MITCHELL JB, COLLINS J, MYERS C: Pharmacological evaluation of intravenous delivery of 5-bromodeoxyuridine to patients with brain tumors. Cancer Res 44: 1702-1705, 1984.

SELDEN JR, DOLBEARE F, CLAIR JH, MILLER JE, MCGETTIGAN K, DIJOHN JA, DYSART GR, DELUCA JG: Validation of a flow cytometric in vitro DNA repair (UDS) assay in rat hepatocytes. Mutat Res 315: 147-167, 1994.

ŠEVC J, DAXNEROVÁ Z, HAŇOVÁ V, KOVAL' J: Novel observations on the origin of ependymal cells in the ventricular zone of the rat spinal cord. Acta Histochem 113: 156-162, 2011.

SPASSKY N, MERKLE FT, FLAMES N, TRAMONTIN AD, GARCIA-VERDUGO JM, ALVAREZ-BUYLLA A: Adult ependymal cells are postmitotic and are derived from radial glial cells during embryogenesis. $J$ Neurosci 25: 10-18, 2005.

TAUPIN P: BrdU immunohistochemistry for studying adult neurogenesis: paradigms, pitfalls, limitations, and validation. Brain Res Rev 53: 198-214, 2007. 
ZHAO M, JANSON LANG AM: Bromodeoxyuridine infused into the cerebral ventricle of adult mice labels nigral neurons under physiological conditions-a method to detect newborn nerve cells in regions with a low rate of neurogenesis. J Neurosci Methods 184: 327-331, 2009. 